\title{
A new strategy for treatment of Anosmia and Ageusia in COVID-19 patients
}

\author{
Asiyeh Hosseini ${ }^{1,}{ }^{*}$, Esmaeil Mirmahdi ${ }^{2}$, and Mina Akbari Moghaddam ${ }^{3}$ \\ ${ }^{1}$ Responsible for Rural Health Services of Dehkool, Hamoon City, Sistan and Baluchistan State, Iran \\ ${ }^{2}$ Lecturer \& Professor Mechanical Engineering, Technical and Vocational University of Golpayegan, Isfahan State, Iran \\ ${ }^{3}$ Responsible for Rural-Urban Health Services of Zahak, Zahak City, Sistan and Baluchistan State, Iran
}

Received 4 September 2020, Accepted 17 November 2020, Published online 17 December 2020

\begin{abstract}
Introduction: The sudden onset of smell and taste loss has been reported as a symptom related to COVID-19. There is new evidence for the loss of smell and taste as a symptom of COVID-19 infection. Objectives: This study aims to examine the influence of coffee on the smell and taste of patients with Covid-19. Methods: In order to investigate the effect of coffee consumption on the taste and smell of COVID-19 patients, we attempted to sample in 20 provinces of the country, COVID-19 patients were separated by gender and underlying and non-underlying disease, doses of 15-20 mg for non-underlying patients and 25-30 mg for underlying patients. The reason for determining this amount of coffee is that every coffee we want to prepare needs a scoop of $14 \mathrm{~g}$ of coffee, which we determine. For some people, it was determined $14 \mathrm{~g}$ and for some people $28 \mathrm{~g}$ of coffee was determined due to consumed in two cups of coffee. Results: Patients with COVID-19 had reversibility on the first and second day. It was clear that there is a small gap between the proportion of recovered patients in the underlying and non-underlying patients, but due to the high volume of observations, this small difference is evident. Discussion: The effectiveness time of coffee is debatable by prescribing its amount. The time efficiency of improving the sense of smell and taste with coffee consumption was lower in non-background patients than in patients. Conclusions: Anosmia and Ageusia are more prevalent in COVID-19 patients. Caffeine in coffee reduced the reversibility of the sense of smell and taste of people with COVID-19. It was concluded that the chances of recovery are higher for those who do not have the underlying disease than for the other groups and for those who suffer from all three types of the underlying disease than for the other groups.
\end{abstract}

Keywords: Anosmia, Ageusia, Coffee, COVID-19, Recovery Time

\section{Introduction}

In December 2019, Coronavirus Disease 2019 (COVID-19) outbreak occurred in Wuhan, Hubei Province, China and spread rapidly throughout China, and then emerged around the world [1-3]. On February 12, 2020, World Health Organization (WHO) named the disease caused by the novel coronavirus as COVID-19 [4]. Clinical evidence has shown that severe acute respiratory syndrome coronavirus 2 (SARS-CoV-2) can be transmitted by person-to-person [1]. Since the outbreak of the COVID-19 pandemic, observations and scientific reports have been accumulating rapidly that sudden anosmia and taste disorders are symptoms associated with the COVID-19 infection $[5,6]$. Recently the WHO has included the loss of smell or taste as a new symptom of COVID-19 infection as have many Health Authorities after a surge of publications and

\footnotetext{
*Corresponding author: asiyehhosseini70@yahoo.com
}

press releases that pointed to anosmia as a potential screening symptom that might contribute to the decision to test suspected cases or guide quarantine instructions. COVID-19 has also been having a major impact on health due to its higher degree of transmissibility, leading to a rapid worldwide dispersion [7]. The main symptoms identified so far are: fever, cough, fatigue, myalgia, arthralgia and dyspnea, which can result in respiratory failure [8]. Nonrespiratory symptoms such as palpitations, abdominal pain, diarrhea, headache and dizziness may precede respiratory symptoms, or come in isolation [9]. It is known that the virus has as target cells that express receptors of the Angiotensin 2 Converting Enzyme (ACE2). ACE2 receptors are expressed predominantly by epithelial cells of the lung, intestine, kidney, heart and blood vessels, the former being the main organ affected by SARS-CoV-2 [10]. However, this does not seem to be the only route of virus entry into the cells, since the liver is quite affected although it does not have many ACE2 receptors [11]. The human brain also shows 


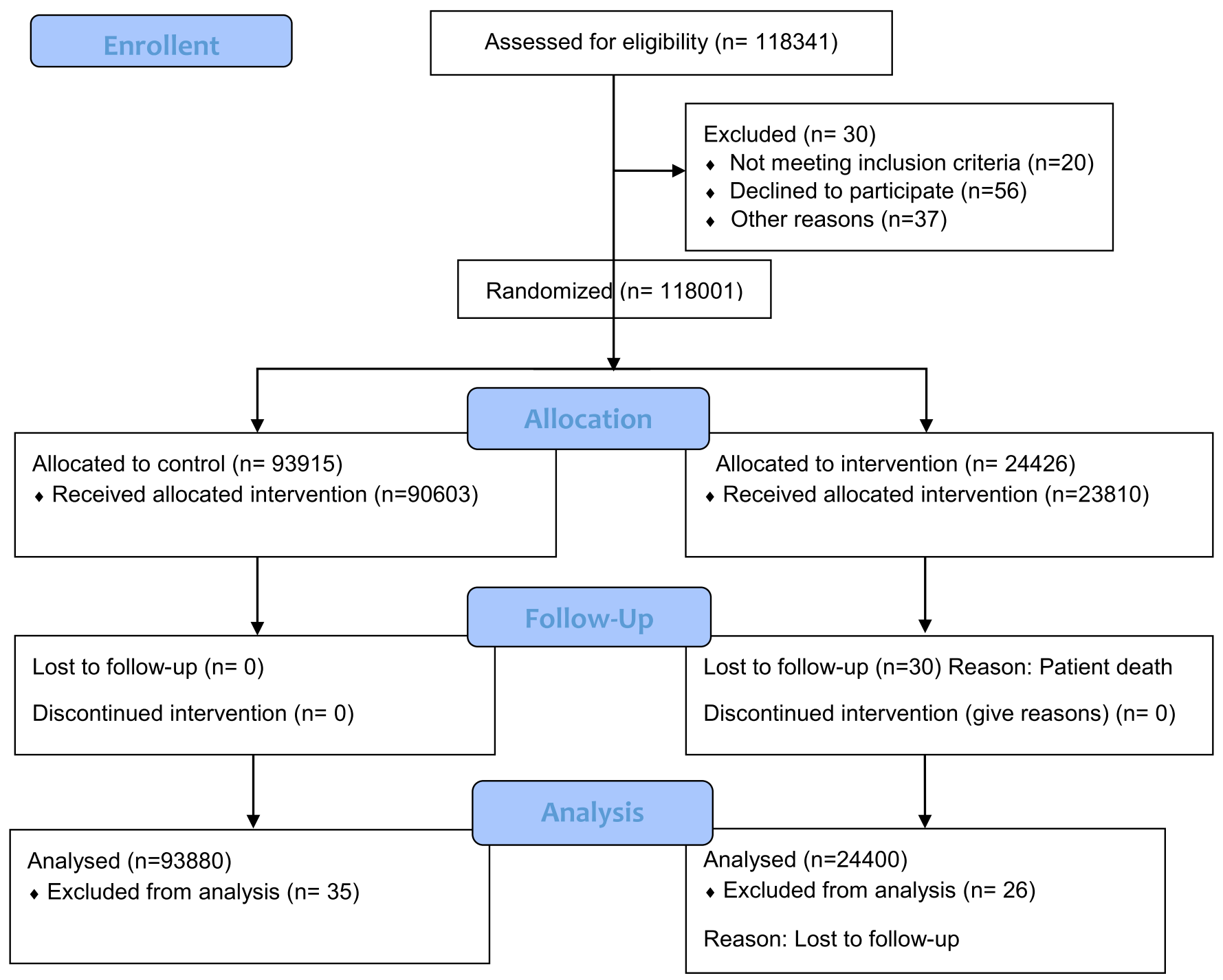

Figure 1. CONSORT flow diagram.

itself as a site with the expression of ACE2 receptors, which seems to justify the neurotropism of SARS-COV-2 by the Central Nervous System (CNS). Studies corroborate the hypothesis of tropism of the coronavirus by the olfactory neuroepithelium and of neurological manifestations in confirmed cases of the disease $[12,13]$. The increase in cases of a sudden loss of the sense of smell (SLoS) noted in medical care during the COVID-19 pandemic motivated the present study. The physiological importance of olfaction in identifying environmental factors and potential threats is so important that the loss of the olfactory sense is related to a reduction in life expectancy, even in individuals without a diagnosis of neurodegenerative disease such as Alzheimer's or Parkinson's disease [14]. The olfactory disorder is a problem already described in most of the countries affected by COVID-19 [15]. In the absence of any comprehensive analysis of the subject, we reviewed the published literature on COVID-19 associated early dysgeusia and anosmia, finding a total of five studies from the European community, China, Italy, USA, and Iran. These yielded a total of 10,847 COVID-19 patients; 8816 (81.27\%), and 8119 (74.85\%) presented with/developed dysgeusia and/or anosmia, respectively indicating these symptoms in almost threequarters of COVID-19 patients. In developing countries like Brazil, COVID-19 testing is restricted to a small portion of patients due to the lack of availability of tests for the entire population. Thus, mild and even moderate cases are not being reported through positive laboratory evidence [5-13]. However, there are knowledge gaps.

The objective of this systematic review was to examine the effect of coffee on Anosmia and Ageusia in COVID-19 Patients. The main reason for choosing coffee as a laboratory material in the present study is that due to its caffeine content, coffee can affect the sense of smell and taste of 
COVID-19 patients. But substances such as caffeinated beverages have not been selected because they can be harmful to people with diabetes.

\section{Method}

It takes at least 30-45 days for reversibility of the sense of smell and taste. In the present study, according to experiments, caffeine of coffee in different groups has had significant effects. In order to investigate the effect of coffee consumption on the taste and smell of COVID-19 patients, we attempted to sample in 20 provinces of the country, COVID-19 patients were separated by gender and underlying and non-underlying disease, doses of 15-20 mg for nonunderlying patients and 25-30 mg for underlying patients. In this study, field patients are divided into the following four groups: (1) Diabetes and hypertension; (2) Heart disease and hypertension; (3) Heart disease and diabetes; (4) Heart disease, hypertension and diabetes. The Consort flow diagram is also shown in Figure 1.

Finally, it was investigated the issue of patients' recovery or non-recovery according to their recovery time and the results were recorded. In the following, it will examine the observations in descriptive statistics and inferential statistics. To describe the observations, first, we plotted the bar graphs of the number of recovered and non-recovered people by those who consumed coffee and those who did not consume coffee (Fig. 2).

Figure 2 indicated that how coffee consumption has an influence on improving people. However, in order to provide a further distinction, in reporting the number of recoveries, it was also used the type of underlying and without the underlying disease (Fig. 3). Again, like Figure 2, we observed that how much coffee consumption plays a role in improving people. Also, people without non-underlying diseases are more common than other people.

Diabetes and hypertension are also the most common underlying diseases. Among them, patients that have been affected by all three types of underlying diseases have the lowest frequency. In order to make better use of the information in these graphs, the following table, it was extracted the total number of patients and the proportion of recoveries for people who drank coffee separately into groups of underlying and without underlying patients.

The smallest rate of recovery is 0.966 and is related to patients who have all three underlying diseases; also the highest rate is related to people who do not have underlying diseases. The ratio of those who have not consumed coffee is equal to 0.014 and is related to people who have all three underlying diseases. In the first step, we examined the effectiveness of coffee consumption in improving the sense of smell and taste by a group of underlying and without underlying patients. The findings are shown in Table 1. It should be noted that we examined the gender factor separately in all of the following inferences.

In Table 1, it uses Chi-squared tests such as Pearson ChiSquare, Likelihood Ratio, Fisher's Exact Test, and Linearby-Linear Association for contingency tables. By using

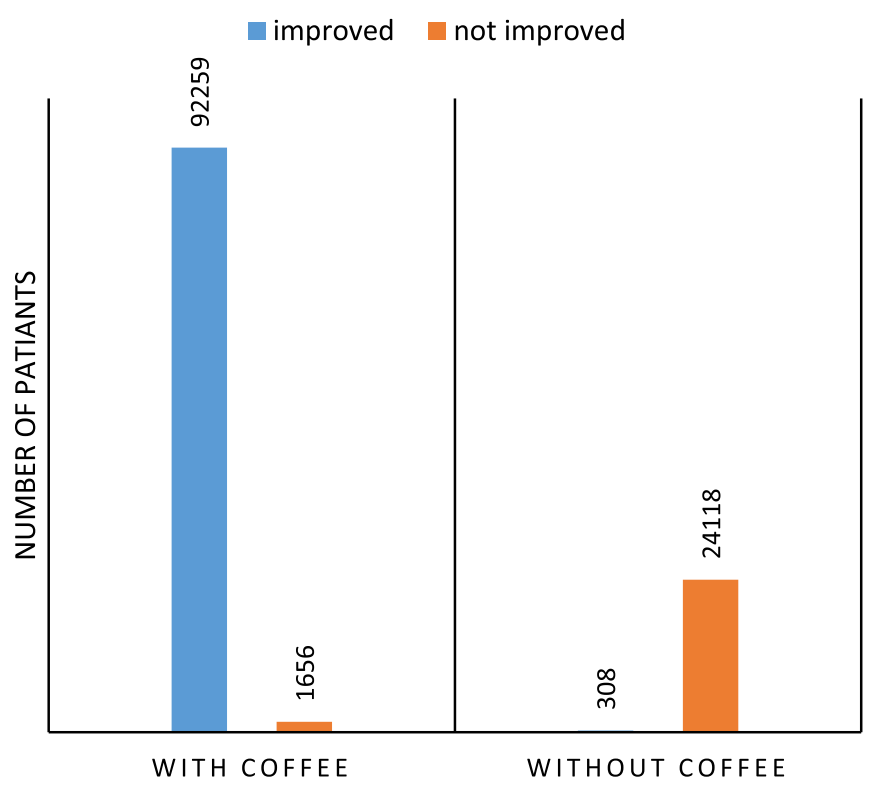

Figure 2. Number of recovered and unhealed if consumed and not consumed coffee.

two columns on the right that show significance, the initial assumption can be judged. It has been calculated significant values in these two columns by using two methods of asymptote and Monte Carlo. Here, we observed that the significance is equal to 0.000 in all cases. Therefore, for all test methods, in all groups of underlying and non-underlying patients, we reject the initial assumption at the level of $\alpha=0.01$ and we accept the alternative assumption based on the effectiveness of coffee consumption in improving patients.

The last line of this table was marked by Total, it examines the positive effect of coffee consumption on the recovery of patients, regardless of whether the patient is underlying and non-underlying. In this line, we also observed the effectiveness of coffee consumption in improving patients.

We also examined whether underlying and non-underlying diseases are effective in the recovery of individuals or not, for this reason, it was extracted in Table 2. In this table, we observed that the type of underlying disease is effective in improving patients. Although in Table 3, there is a small gap between the proportion of recovered patients in the underlying and non-underlying patients, due to the high volume of observations, this small difference is evident. Carefully in Table 3, we find that the chances of recovery are higher for those who do not have the underlying disease than for the other groups and for those who suffer from all three types of the underlying disease than for the other groups.

\section{Discussion}

The effectiveness of coffee in this article has been reported for $5-7 \mathrm{~h}$ for non-fixeld patients and between 

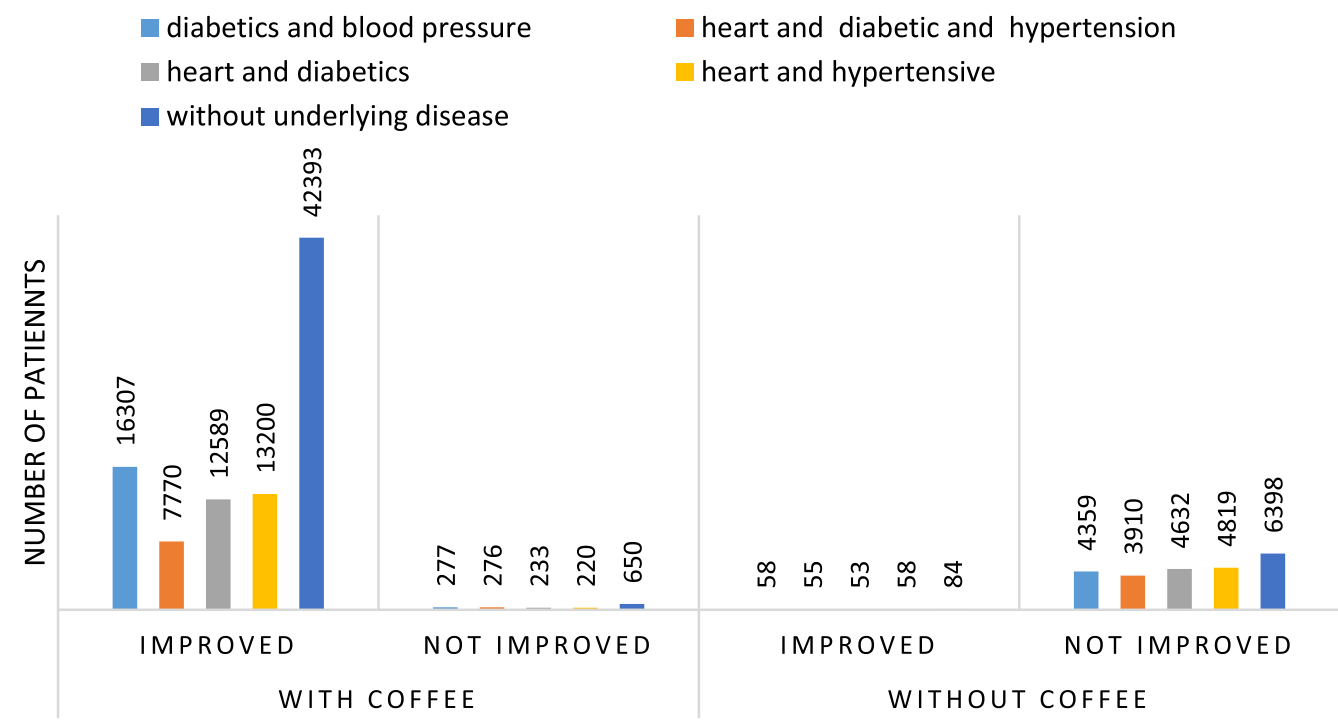

Figure 3. Number of recovered and non-recovered in case of consuming and not consuming coffee, separately for underlying without underlying patients.

Table 1. Chi-Square tests to evaluate the effectiveness of coffee consumption in improving patients' sense of smell and taste by a group of underlying and without underlying patients.

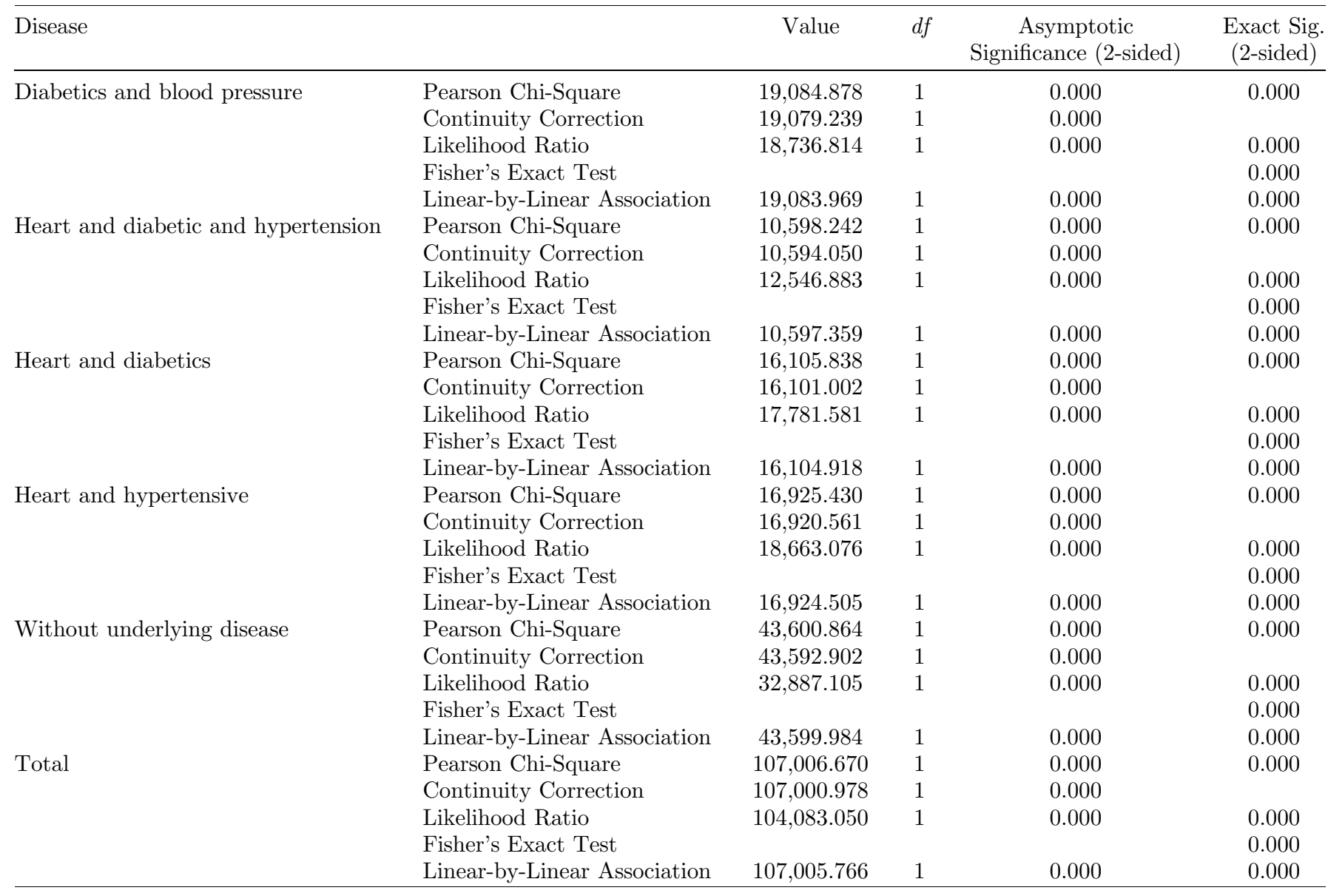


Table 2. Chi-Square tests to evaluate the effect of underlying and non-underlying disease type on improving patients' sense of smell and taste.

\begin{tabular}{lccc}
\hline & Value & $d f$ & Asymptotic significance (2-sided) \\
\hline Pearson Chi-Square & 3589.678 & 4 & 0.000 \\
Likelihood Ratio & 3589.482 & 4 & 0.000 \\
Linear-by-linear association & 1388.499 & 1 & 0.000 \\
No of valid cases & 118,341 & & \\
\hline
\end{tabular}

Table 3. The total number of patients and the proportion of recovered patients by underlying are for people who have consumed coffee.

\begin{tabular}{|c|c|c|c|c|c|}
\hline & & & & \multicolumn{2}{|c|}{ Consumption } \\
\hline & & & & With coffee $(\%)$ & Without coffee $(\%)$ \\
\hline \multirow[t]{7}{*}{ Disease } & Diabetics and blood pressure & Improvement & Improved & 98.3 & 1.3 \\
\hline & \multirow[t]{2}{*}{ Heart and diabetic and hypertension } & \multirow[t]{2}{*}{ Improvement } & Improved & 96.6 & 1.4 \\
\hline & & & Not improved & 3.4 & 98.6 \\
\hline & Heart and diabetics & Improvement & Improved & 98.2 & 1.1 \\
\hline & Heart and hypertensive & Improvement & Not improved & 1.6 & 98.8 \\
\hline & \multirow[t]{2}{*}{ Without underlying disease } & \multirow[t]{2}{*}{ Improvement } & Improved & 98.5 & 1.3 \\
\hline & & & Not improved & 1.5 & 98.7 \\
\hline
\end{tabular}

2 and 4 days for underlying patients depending on the type of disease. Outpatients in this trial have a better recovery process, but all efforts were made for outpatients to ensure the recovery process. It was difficult for them to suffer from the disease, except for Corona, and with coffee, the process of healing their sense of smell and taste returned dramatically. Coffee has a specific amount of consumption, which is $15-20 \mathrm{mg}$ of coffee for non-field patients and $20-30 \mathrm{mg}$ for underlying patients. All results showed that the recovery of non-background patients was higher than the underlying patients, although the underlying patients also had less treatment.

\section{Conclusion}

In the treatment of people with COVID-19, the last step that is reversible is the sense of smell and taste. These patients tend to get a sense of taste and smell as soon as possible. The results of the present experiment indicated that caffeine in coffee reduced the reversibility of the sense of smell and taste of people with COVID-19. The smallest rate of recovery is 0.966 and is related to patients who have all three underlying diseases; also the highest rate is related to people who do not have underlying diseases. The ratio of those who have not consumed coffee is equal to 0.014 and is related to people who have all three underlying diseases. Also, we concluded that the alternative assumption is based on the effectiveness of coffee consumption in improving patients. We also observed the effectiveness of coffee consumption in improving patients. There is little difference between the proportion of improved patients in underlying and non-underlying patients, but due to the high volume of observations, this small difference is evident. So, we understood that the chances of recovery are higher for those who do not have the underlying disease than for the other groups and for those who suffer from all three types of the underlying disease than for the other groups. The effectiveness of coffee has been reported to be $5-7 \mathrm{~h}$ for outpatients and between 2 and 4 days for underlying patients, depending on the type of disease.

\section{Ethics approval and consent to participate}

Ethics committee approval for a case report was considered. The patient gave her written informed consent to the medical procedures used for treatment purposes

\section{Conflicts of interest}

The authors declare no conflicts of interest.

\section{Acknowledgments}

This study was conducted by a team of four people who came together to develop anticancer devices before the outbreak of coronary heart disease. They were building this device initially by ultrasound waves under the supervision of Eng. Mr. Mirmahdi. But after the outbreak of this disease, their goal and attention were concentrated on how to this disease. The authors would like to thank all Iranian 
health workers, Eng. Mr. Mehdi Zarei, Ph.D. student of natural resources at American Michigan University, for the efforts and sacrifices they are making during this serious health crisis. Also, the authors wish to thank patients for their participation and kind cooperation.

\section{Research Limitations}

One of the limitations of this study is that it had no tendency to participate in a group of older patients. It was also difficult for them to accept that their sense of smell and taste was recovered by consuming coffee, which they achieved after consuming this important event. Finding patients with an underlying disease was difficult in months of effort. Spending a lot of time-consuming coffee and training it to prepare and type of consumption at some ages, which were generally over the age of 45 , made the article more time-consuming and lengthier.

\section{References}

1. Bagheri SHR, Asghari AM, Farhadi M, Shamshiri AR, Kabir A, Kamrava SK (2020), Coincidence of COVID-19 epidemic and olfactory dysfunction outbreak. medRxiv. http://dx.doi.org/10.1101/2020.03.23.20041889.

2. Baig AM, Khaleeq A, Ali U, Syeda H (2020), Evidence of the COVID-19 virus targeting the CNS: tissue distribution, hostvirus interaction, and proposed neurotropic mechanisms. ACS Chem Neurosci. https://doi.org/10.1021/acschemneuro. 0c00122.

3. Bavishi C, Maddox TM, Messerli FH (2019), Coronavirus disease 2019 (COVID-19) infection and renin angiotensin system blockers. JAMA Cardiol 2020. https://doi.org/ 10.1001/jamacardio.2020.1282.

4. Giacomelli A, Pezzati L, Conti F (2020), Self-reported olfactory and taste disorders in SARS-CoV-2 patients: a cross-sectional study. Clin Infect Dis. https://doi.org/ $10.1093 / \mathrm{cid} / \mathrm{ciaa} 330$
5. Guan WJ, Ni ZY, Hu Y, Liang WH, Ou CQ, He JX. 2020. Clinical characteristics of coronavirus disease 2019 in China. N Engl J Med 382, 1708-1720. https://doi.org/10.1056/ NEJMoa2002032.

6. Lechien JR, Chiesa-Estomba CM, De Siati DR. 2020. Olfactory and gustatory dysfunctions as a clinical presentation of mildtomoderate forms of the coronavirus disease (COVID-19): a multicenter European study. Eur Arch Otorhinolaryngol. https://doi.org/10.1007/s00405-020-05965-1.

7. Li Q, Guan X, Wu P, Wang X, Zhou L, Tong Y (2020), Early transmission dynamics in Wuhan, China, of novel coronavirus-infected pneumonia. N Engl J Med 382, 1199-1207. https://doi.org/10.1056/NEJMoa2001316.

8. Lüers J-C, Klußmann JP, Guntinas-Lichius O (2020), Die Covid-19-Pandemie und das HNO-Fachgebiet: Worauf kommt es aktuellan? Laryngo-Rhino-Otologie 99, 287-291.

9. Mao L, Wang M, Chen S, He Q, Chang J, Hong C (2020), Neurological manifestations of hospitalized patients with COVID-19 in Wuhan, China: a retrospective case series study. medRxiv. https://doi.org/10.1001/jamaneurol.2020.1127.

10. Park SE (2020), Epidemiology, virology, and clinical features of severe acute respiratory syndrome coronavirus-2 (SARS-CoV-2; Coronavirus Disease-19). Clin Exp Pediatr 63, 119-124.

11. Steardo L, Steardo L, Zorec R, Verkhratsky A (2020), Neuroinfection may potentially contribute to pathophysiology and clinical manifestations of COVID-19. Acta Physiol. https://doi.org/10.1111/apha.13473.

12. World Health Organization (2020), Coronavirus disease 2019 (COVID-19) situation Report-104. https://www.who. int/docs/default-source/coronaviruse/situation-reports/ 20200503-COVID-19-sitrep-104.pdf?sfvrsn $=53328 f 46 \_2$.

13. World Health Organization (2020), https://www.who.īnt/dg/ speeches/detail/whodirector-general-s-remarks-at-the-mediabriefing-on-2019-ncov-on-11-februarv-2020. https://www. who.int/docs/default-source/coronaviruse/situation-reports/ 20200503-COVID-19-sitrep-104.pdf?sfvrsn $=53328 f 462$.

14. Wilson RS, Yu L, Bennett DA (2011), Odor identification and mortality in old age. Chem Sens 36, 63-67.

15. Xu K, Lai XQ, Liu Z (2019), Suggestion for prevention of 2019 novel coronavirus infection in otolaryngology head and neck surgery medical staff. https://doi.org/10.3760/cma.j. issn.1673-0860.2020.0001. 